\title{
Inelastic black hole scattering from charged scalar amplitudes
}

\author{
Andrés Luna, ${ }^{a}$ Isobel Nicholson, ${ }^{b, c}$ Donal $\mathrm{O}^{\prime}$ Connell ${ }^{b, c}$ and Chris D. White ${ }^{d}$ \\ ${ }^{a}$ School of Physics and Astronomy, University of Glasgow, \\ G12 8QQ, Scotland, U.K. \\ ${ }^{b}$ Higgs Centre for Theoretical Physics, School of Physics and Astronomy, \\ The University of Edinburgh, \\ Edinburgh EH9 3JZ, Scotland, U.K. \\ ${ }^{c}$ Kavli Institute for Theoretical Physics, University of California, \\ Santa Barbara, CA 93106-4030, U.S.A. \\ ${ }^{d}$ Centre for Research in String Theory, School of Physics and Astronomy, \\ Queen Mary University of London, \\ 327 Mile End Road, London E1 4NS, U.K. \\ E-mail: a.luna-godoy.1@research.gla.ac.uk, i.nicholson@sms.ed.ac.uk, \\ donal@ph.ed.ac.uk, christopher.white@qmul.ac.uk
}

ABSTRACT: We explain how the lowest-order classical gravitational radiation produced during the inelastic scattering of two Schwarzschild black holes in General Relativity can be obtained from a tree scattering amplitude in gauge theory coupled to scalar fields. The gauge calculation is related to gravity through the double copy. We remove unwanted scalar forces which can occur in the double copy by introducing a massless scalar in the gauge theory, which is treated as a ghost in the link to gravity. We hope these methods are a step towards a direct application of the double copy at higher orders in classical perturbation theory, with the potential to greatly streamline gravity calculations for phenomenological applications.

KeYwords: Scattering Amplitudes, Black Holes, Classical Theories of Gravity

ArXiv EPrint: 1711.03901 


\section{Contents}

1 Introduction 1

2 Review 3

2.1 Review of BCJ 3

2.2 Classical gravitational scattering 5

3 Charged scalar amplitudes $\quad 7$

3.1 The scattering amplitude 8

$\begin{array}{lll}3.2 & \text { Large mass expansion } & 10\end{array}$

4 Removing the dilaton $\quad 13$

$\begin{array}{lll}4.1 & \text { Double copy and massive amplitudes } & 13\end{array}$

$\begin{array}{ll}4.2 & \text { Dilatons in diagrams } \\ \end{array}$

$\begin{array}{lll}4.3 & \text { Example at four points } & 16\end{array}$

$\begin{array}{lll}4.4 & \text { Inelastic scattering } & 17\end{array}$

$\begin{array}{ll}\text { 4.5 Relation to classical field computation } & 18\end{array}$

$\begin{array}{lll}5 & \text { Discussion and conclusions } & 19\end{array}$

\section{Introduction}

General Relativity is a spectacularly successful description of gravitational processes. It is also celebrated for its great beauty. However, the perturbative expansion of the EinsteinHilbert Lagrangian about Minkowski space is a less well-beloved aspect of Einstein's theory. Because of the presence of the metric and its inverse, as well as the square root of the determinant of the metric, the perturbative expansion contains an infinite number of terms, each of which corresponds to a complicated Feynman rule. This complexity makes calculation difficult.

Gradually we have realised that a surprising approach to gravitational perturbation theory about Minkowski space has the potential to greatly improve this situation. The basis of the idea goes back to the early days of string theory, when Kawai, Lewellen and Tye (KLT) realised [1] that closed string amplitudes can be obtained from open string amplitudes. In the field theory limit, this means that graviton scattering amplitudes may be obtained from knowledge of gauge boson scattering amplitudes, and of the KLT relations.

One disadvantage of the KLT connection between gauge theory and gravity is that the KLT relations themselves are quite complicated, especially for processes involving many gravitons. They are also restricted to tree level scattering amplitudes, so that their applicability to loop processes is necessarily indirect. Fortunately, another perspective on the 
connection between gauge theory and gravity, known as the Bern, Carrasco and Johansson (BCJ) double copy, removes both of these disadvantages $[2,3]$. The double copy is based on a simple rearrangement of Yang-Mills amplitudes which has a distinctly group theoretic feel. It also has an immediate application to loop diagrams.

To date, the double copy has been used primarily to understand the quantum structure of gravity. But there is also considerably motivation to understand classical processes in General Relativity to high precision. This motivation comes from future plans for high precision gravitational wave observatories, such as LISA and the Einstein telescope. Does the double copy provide a route to a refined understanding of radiative processes involving black holes? In fact, scattering amplitudes have already been used to extract information about the classical scattering processes in gravity. One direction is to determine the effective potential between objects deduced from two-to-two amplitudes, which has been studied in a series of remarkable papers which build on the full suite of amplitudes tools [4-10], see also [11] for a recent pedagogical introduction. There is clearly great scope for further work in this area [12].

However, the double copy is set up to compute graviton scattering amplitudes. Perhaps it may be possible to directly compute the classical gravitational wave spectrum in a scattering process directly from the double copy? Indeed, the situation seems to be very positive. The structure of the double-copy is reflected in a class of solutions of the Einstein equations, known as Kerr-Schild solutions. These simple, symmetric spacetimes are associated with similarly simple and symmetrical exact solutions of the Yang-Mills equations in a classical manifestation of the double copy [13-20]. Similarly, perturbative spacetimes can be constructed order-by-order in a manner which directly manifests the double copy [21]. But it was still a wonderful surprise to see genuine gravitational scattering, with the production of gravitational radiation, emerge in a remarkably simple form from the double copy as shown recently by Goldberger and Ridgway [22], see also [23].

One disadvantage of the Goldberger-Ridgway calculation is the presence of unwanted fields in the classical theory. In the double copy it is a simple fact that the graviton naturally comes along with two extra fields: a scalar dilaton and an antisymmetric tensor known as the axion. In any given calculation, we can hope to switch the dilaton and axion off, but typically in the simplest cases they will be present. Indeed, in the simplest perturbative construction of a spacetime containing a point-like singularity using the double copy [21], the dilaton couples to the singularity with strength proportional to the gravitational mass of the singularity. The spacetime is therefore a JNW naked singularity [24] rather than the Schwarzschild solution. As a consequence, the objects scattering in the GoldbergerRidgway calculation were JNW singularities.

Another interesting aspect of reference [23] was how the authors implemented the double copy. Rather than following the standard prescription from scattering amplitudes, they replaced Yang-Mills colour factors with the kinematic part of the Yang-Mills three point vertex. This is remarkable in view of the fact that it is well-known [25-37] that the four point vertex in gauge theory is important for colour-kinematics to work in general.

The goal of this paper is to understand classical inelastic gravitational scattering from the point of view of scattering amplitudes, given that it is in this context that the double 
copy has its most well-understood form. In particular, we will show how to obtain gravitational radiation by copying a Yang-Mills amplitude. Working at lowest order, it is sufficient to consider the scattering of a pair of scalar particles, which represent non-spinning (Schwarzschild) black holes. We will show explicitly how the Goldberger-Ridgway approach can be related to scattering amplitudes, and comment on a particular simplification which occurs in the double copy in this specific context. Furthermore, the amplitude approach can be used to remove the contribution of the dilaton. To this end, we build on the recent work of Johansson and Ochirov [38], who obtained pure gravity as a double copy.

We hope that our results represent a step toward an application of the double copy in more detailed calculations of gravitational phenomena. Evidently the double copy has the capacity to determine genuine perturbations to the metric about Minkowski space resulting from physical dynamical processes. The technical simplicity of the double copy could greatly streamline the calculation of phenomenologically relevant quantities if it can be applied to physically relevant processes.

The structure of our paper is as follows. In section 2 we recall salient details regarding BCJ duality and the double copy, as well as reviewing the classical scattering calculations of refs. [22]. In section 3 we show how these results can be reproduced using scattering amplitudes, which will involve a detailed discussion of taking appropriate kinematic limits to make this equivalence manifest. In section 4 , we demonstrate explicitly how the dilaton can be removed to generate results in pure gravity, building on the ideas of [38]. Finally, we discuss our results and conclude in section 5 .

\section{Review}

To keep the article self-contained, we open with a review of the BCJ story: colourkinematics duality and the double copy. We have also included a brief review of reference [23] which will be important for the remainder of the paper.

\section{$2.1 \quad$ Review of BCJ}

The essence of BCJ is an understanding of how gauge invariance works in the scattering amplitudes of Yang-Mills theory. We can always choose to write these amplitudes as a sum over Feynman-like diagrams with three-point vertices. If the set $\Gamma$ contains all of these diagrams with $n$ external points, then the $n$-point amplitude is

$$
\mathcal{A}=\sum_{i \in \Gamma} \frac{c_{i} n_{i}}{d_{i}}
$$

where $d_{i}$ is the Feynman propagator denominator associated with graph $i$, while $c_{i}$ is the Yang-Mills colour factor corresponding to the diagram and $n_{i}$ is the kinematic numerator of the graph. Notice that only the kinematic numerator $n_{i}$ depends on the polarisations of the particles. Let us choose one particle, say particle 1, and replace its polarisation vector $\epsilon_{1}$ by its momentum $p_{1}$. In that case gauge invariance requires that the amplitude $\mathcal{A}=0$. However, it is not the case that all the numerators $n_{i}=0$. Rather, the identity $\mathcal{A}=0$ follows from a cancellation among the distinct diagrams. This is only possible because the 
colour factors $c_{i}$ are not all independent. Instead, they obey Jacobi identities which arise from pure group theory.

It is also the case that the $n_{i}$ are not all uniquely defined. Indeed, there is a large space of numerators which have the property that (2.1) is a valid expression for the YangMills amplitude. The idea of BCJ is then to pick very special numerators which have the property that whenever graphs $\alpha, \beta$ and $\gamma$ are such that the colour factors satisfy a Jacobi identity,

$$
c_{\alpha} \pm c_{\beta} \pm c_{\gamma}=0
$$

then the kinematic numerators satisfy the same identity,

$$
n_{\alpha} \pm n_{\beta} \pm n_{\gamma}=0
$$

Notice that we have allowed for the possibility that there may be positive and negative signs in the Jacobi identity; whatever these signs are, they must be in common between the colour and kinematic identities. We also require that the kinematic numerators satisfy the same antisymmetries as the colour factors. These requirements are known as colourkinematics duality, because both colour and kinematics have the same algebraic properties.

The reason for making this choice is that we may now construct a new amplitude which must be gauge invariant and local:

$$
\mathcal{M}=\sum_{i \in \Gamma} \frac{n_{i} n_{i}}{d_{i}}
$$

Comparing to our previous gauge amplitude, we have replaced the colour factors $c_{i}$ with a second copy of the kinematic numerators, $n_{i}$. For this reason, equation (2.4) is known as the double copy formula. The quantity $\mathcal{M}$ is gauge invariant because, if we replace the polarisation vector $\epsilon_{1}$ with $p_{1}$ in, say, the left $n_{i}$ factors, then $\mathcal{M}=0$. The identity must follow from precisely the same algebra as in gauge invariance of the Yang-Mills amplitude. Similarly we could replace the polarisation vector with the momentum in just the right $n_{i}$ factor. Locality is assured because we have included precisely the correct Feynman denominators for a local field theory. Therefore $\mathcal{M}$ is a scattering amplitude of some kind.

It is straightforward to see that $\mathcal{M}$ is an amplitude in a theory of gravity. To see this, notice that for each particle, $\mathcal{M}$ is linear in the outer product $\epsilon_{i}^{\mu} \epsilon_{i}^{\nu}$ of polarisation vectors. We may decompose this outer product into irreducible representations of the little group. The symmetric, traceless tensor is the polarisation tensor of a gravitational wave. Other kinds of particles are present: the trace term in the tensor product decomposition corresponds to a massless scalar particle which is known as the dilaton, while the antisymmetric tensor is known as the axion. The presence of these states is a natural feature of the double copy. Since in the double copy, the graviton, the axion and the dilaton all emerge from a tensor product decomposition of one matrix, it is useful to give them a collective name: we will refer to them as the product graviton. ${ }^{1}$

\footnotetext{
${ }^{1}$ The term fat graviton was used in ref. [21] for this quantity. Here we wish to avoid ambiguity due to a similar term being used elsewhere in the literature.
} 
More generally, we can allow for two different choices of $n_{i}$ in the double copy, for example numerators of different gauge theories:

$$
\mathcal{M}=\sum_{i \in \Gamma} \frac{n_{i} \tilde{n}_{i}}{d_{i}} .
$$

In this way we can construct amplitudes corresponding to a variety of theories. There is an active programme of research aimed at determining what kinds of gravitational theories can be constructed from the double copy [40-48]. The construction of pure Einstein gravity as a double copy, due to Johansson and Ochirov [38] is a particularly interesting case, and it is one which will play a central role in the present paper.

It is also worth emphasising that the double copy has an immediate extension to loop amplitudes [3]. This fact has led to a wealth of progress in our understanding of the structure of (super)gravity [49-62], most recently including the integrand of the fiveloop, four-point amplitude in maximal supergravity in four dimensions [63]. This last achievement rested on a new general technique [64] for constructing appropriate numerators which we anticipate will be very useful in the future. All-order evidence for the double copy has been obtained in special kinematic limits [65-70].

The double copy rests on colour-kinematics duality, which hints at the existence of a new kind of symmetry in gravity — a kinematic symmetry which controls the structure of the kinematic numerators. This kinematic algebra remains mostly mysterious, except in the context of the self-dual theory [71] and the nonlinear sigma model [72]. But in spite of the slow progress in our understanding of the kinematic algebra, the double copy provides a new way of understanding the symmetry structure of supergravity as following from symmetries of Yang-Mills theory [73-79].

\subsection{Classical gravitational scattering}

In this section, we review the work of Goldberger and Ridgway [22], who computed the gravitational radiation emitted during the inelastic scattering of two JNW singularities using a method based on the double-copy. Thus, their computation began in the context of gauge theory. Specifically, they considered classical, coloured point particles with positions $x_{i}(\tau)$ and colours $c_{i}^{a}(\tau)$ and masses $m_{i}$ interacting through a gauge field $A_{\mu}^{a}$. If we denote the coupling by $g$ and let $F_{\mu \nu}$ be the gauge field strength tensor, the classical equations defining the system are

$$
\begin{aligned}
D^{\mu} F_{\mu \nu}^{a} & =g \sum_{i} \int d \tau c_{i}^{a}(\tau) \frac{d x_{i}^{\nu}(\tau)}{d \tau} \delta^{(d)}\left(x-x_{i}(\tau)\right), \\
m_{i} \frac{d^{2} x_{i}^{\mu}(\tau)}{d \tau^{2}} & =g F^{a \mu \nu} c_{i}^{a}(\tau) \frac{d x_{i \nu}(\tau)}{d \tau} \\
\frac{d c_{i}^{a}(\tau)}{d \tau} & =g f^{a b c} \frac{d x_{i}^{\mu}}{d \tau} A_{\mu}^{b}\left(x_{i}(\tau)\right) c_{i}^{c}(\tau) .
\end{aligned}
$$

When the scattering angle is small, one can solve these equations order-by-order in perturbation theory. At zeroth order, the particles move on straight-line trajectories, with 
constant velocity $v_{i}^{\mu}$ and constant colour:

$$
\begin{aligned}
x_{i}^{\mu}(\tau) & \simeq b_{i}^{\mu}+v_{i}^{\mu} \tau, \\
c_{i}^{a}(\tau) & \simeq c_{i}^{(0) a} .
\end{aligned}
$$

Interactions correct these expressions, leading to perturbative expansions of the positions, the colours, and indeed of the field. We will indicate terms arising at the $n$th order of perturbation theory with a superscript in brackets:

$$
\begin{aligned}
x_{i}^{\mu}(\tau) & =x_{i}^{(0) \mu}(\tau)+x_{i}^{(1) \mu}(\tau)+\cdots, \\
c_{i}^{a}(\tau) & =c_{i}^{(0) a}+c_{i}^{(1) a}(\tau)+\cdots, \\
A_{\mu}^{a}(x) & =A_{\mu}^{(0) a}(x)+A_{\mu}^{(1) a}(x)+\cdots .
\end{aligned}
$$

With this setup, it is a mechanical task to perturbatively solve the equations of motion to any desired accuracy. We are interested in radiation emitted during a collision, which requires us to compute $A_{\mu}^{(1) a}(x)$. The result is

$$
\begin{aligned}
& k^{2} A^{(1) a \mu}(k)=g^{3} \int{ }^{\top} q_{1}{ }^{\top} q_{2} \delta\left(k-q_{1}-q_{2}\right) \delta\left(q_{1} \cdot v_{1}\right) e^{i q_{1} \cdot b_{1}} \delta\left(q_{2} \cdot v_{2}\right) e^{i q_{2} \cdot b_{2}} \\
& \times\left\{\frac{c_{1}^{(0) a}}{m_{1}} \frac{c_{1}^{(0)} \cdot c_{2}^{(0)}}{k \cdot v_{1} q_{2}^{2}}\left[-v_{1} \cdot v_{2}\left(q_{2}^{\mu}-\frac{k \cdot q_{2}}{k \cdot v_{1}} v_{1}^{\mu}\right)+k \cdot v_{1} v_{2}^{\mu}-k \cdot v_{2} v_{1}^{\mu}\right]\right. \\
& \left.+\frac{i f^{a b c} c_{1}^{b} c_{2}^{c}}{q_{1}^{2} q_{2}^{2}}\left[2 k \cdot v_{2} v_{1}^{\mu}-v_{1} \cdot v_{2} q_{1}^{\mu}+v_{1} \cdot v_{2} \frac{q_{1}^{2}}{k \cdot v_{1}} v_{1}^{\mu}\right]+(1 \leftrightarrow 2)\right\} .
\end{aligned}
$$

Of course, our main focus is not gauge radiation but rather the gravitational radiation obtained via the double copy. Goldberger and Ridgway implemented the double copy as a set of replacement rules:

$$
\begin{aligned}
c_{i}^{(0) a} & \rightarrow m_{i} v_{i}^{\mu}, \\
i f^{a b c} & \rightarrow \frac{1}{2} \Gamma^{\mu \nu \rho}\left(q_{1}, q_{2}, q_{3}\right) .
\end{aligned}
$$

In the latter replacement, the quantity $\Gamma$ is proportional to the Yang-Mills three point amplitude, while the three momenta $q_{1}, q_{2}$ and $q_{3}$ are momenta associated with the lines with colours $a, b$ and $c$. Specialising to the two particle case, the result is a perturbative "product" graviton given by

$$
\begin{aligned}
& k^{2} H^{(1) \mu \nu}(k)=-\frac{m_{1} m_{2}}{8 m_{\mathrm{pl}}^{3(d-2) / 2}} \int \tau q_{1} \tau q_{2} \delta\left(k-q_{1}-q_{2}\right) \delta\left(q_{1} \cdot v_{1}\right) e^{i q_{1} \cdot b_{1}} \delta\left(q_{2} \cdot v_{2}\right) e^{i q_{2} \cdot b_{2}} \\
& {\left[\frac{v_{1} \cdot v_{2}}{q_{2}^{2} k \cdot v_{1}} v_{1}^{\nu}\left\{v_{1} \cdot v_{2}\left(\frac{1}{2}\left(q_{2}-q_{1}\right)^{\mu}-\frac{k \cdot q_{2}}{k \cdot v_{1}} v_{1}^{\mu}\right)+k \cdot v_{2} v_{1}^{\mu}-k \cdot v_{1} v_{2}^{\mu}\right\}\right.} \\
& +\frac{2 k \cdot v_{2} v_{1}^{\nu}-2 k \cdot v_{1} v_{2}^{\nu}+v_{1} \cdot v_{2}\left(q_{2}-q_{1}\right)^{\nu}}{2 q_{1}^{2} q_{2}^{2}}\left(2 k \cdot v_{2} v_{1}^{\mu}-v_{1} \cdot v_{2} q_{1}^{\mu}+\frac{v_{1} \cdot v_{2} q_{1}^{2}}{k \cdot v_{1}} v_{1}^{\mu}\right) \\
& +(1 \leftrightarrow 2)]
\end{aligned}
$$


It was demonstrated in reference [23], by direct calculation, that this product graviton encodes the gravitational radiation emitted in the scattering of two JNW singularities.

For our purposes, it is helpful to exploit the symmetry in particles 1 and 2 to slightly rewrite $H^{(1) \mu \nu}(k)$ in a manner which makes gauge invariance more manifest. To that end, we introduce the vectors

$$
\begin{aligned}
P_{12}^{\mu} & \equiv k \cdot v_{1} v_{2}^{\mu}-k \cdot v_{2} v_{1}^{\mu}, \\
Q_{12}^{\mu} & \equiv\left(q_{1}-q_{2}\right)^{\mu}-\frac{q_{1}^{2}}{k \cdot v_{1}} v_{1}^{\mu}+\frac{q_{2}^{2}}{k \cdot v_{2}} v_{2}^{\mu},
\end{aligned}
$$

which are gauge invariant in the sense that $P_{12} \cdot k=0=Q_{12} \cdot k$. The product graviton can be written as

$$
\begin{aligned}
k^{2} H^{(1) \mu \nu}(k)= & -\frac{m_{1} m_{2}}{8 m_{\mathrm{pl}}^{3(d-2) / 2}} \int d q_{1} d q_{2} \delta\left(k-q_{1}-q_{2}\right) \delta\left(q_{1} \cdot v_{1}\right) e^{i q_{1} \cdot b_{1}} \delta\left(q_{2} \cdot v_{2}\right) e^{i q_{2} \cdot b_{2}} \times \\
& {\left[\frac{P_{12}^{\mu} P_{12}^{\nu}}{q_{1}^{2} q_{2}^{2}}+\frac{v_{1} \cdot v_{2}}{2 q_{1}^{2} q_{2}^{2}}\left(Q_{12}^{\mu} P_{12}^{\nu}+Q_{12}^{\nu} P_{12}^{\mu}\right)+\frac{\left(v_{1} \cdot v_{2}\right)^{2}}{4}\left(\frac{Q_{12}^{\mu} Q_{12}^{\nu}}{q_{1}^{2} q_{2}^{2}}-\frac{P_{12}^{\mu} P_{12}^{\nu}}{\left(k \cdot v_{1}\right)^{2}\left(k \cdot v_{2}\right)^{2}}\right)\right] . }
\end{aligned}
$$

A number of questions arise from this calculation, such as:

1. Are the double-copy replacement rules in equations (2.13) the same as the BCJ rules, or a replacement for them? What about colour-kinematics duality?

2. Can we find a straightforward mechanism for removing the dilaton pollution in the calculation?

To address these questions, we find it convenient to reformulate the black hole scattering calculation as a scattering amplitude.

\section{Charged scalar amplitudes}

Our aim is to recast the emission of gravitational radiation from a pair of scattering particles, in terms of a scattering amplitude calculation. Amplitudes have the advantage that the application of the double copy is well-established, as is the possibility of removing unwanted dilaton contributions. To this end, we must first decide what scattering amplitude to calculate. We will begin in Yang-Mills theory, given that we wish to obtain the gravity result using the double copy. The simplest possible candidate is then a five-point amplitude, corresponding to the incoming / outgoing particles, plus an additional gluon, as shown in figure 1. The scattering particles themselves, however, need not be gluons. Ultimately, our gravity calculation will describe the scattering of astrophysical objects (e.g. black holes) of arbitrary spin. Thus, we must add additional matter to our pure Yang-Mills theory, whose spin is directly related to the spin of the objects whose scattering we wish to study. Given that our main motivation is to illustrate the double copy and removal of the dilaton, we will restrict ourselves to scalar scattering particles in what follows. 


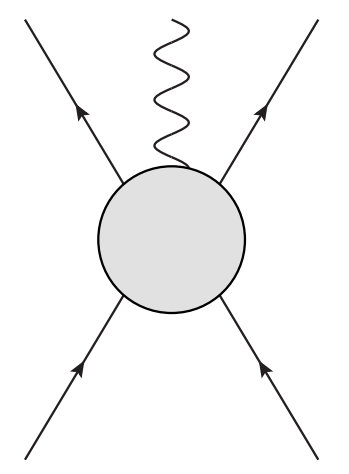

Figure 1. Two particle scattering with the production of radiation. This is the basic structure of diagrams we will be interested in. Note that time is on the vertical axis.

The masses of our two incoming particles need not be the same, so we will consider a gauge field $A_{\mu}^{a}$ coupled to two different massive scalars $\Phi_{i}$ transforming in representations $R_{i}$ of the gauge group. The Lagrangian is simply

$$
\mathcal{L}=-\frac{1}{2} \operatorname{tr} F^{\mu \nu} F_{\mu \nu}+\sum_{i}\left[\left(D_{\mu} \Phi_{i}\right)^{\dagger}\left(D^{\mu} \Phi_{i}\right)-m_{i}^{2}|\Phi|^{2}\right]
$$

A discussion of how the double copy works in this kind of situation can be found in [38, 39]. The double copy of our five point gauge amplitude will be an amplitude in a theory containing gravity, a dilaton, an axion, and the scalar fields. ${ }^{2}$

The goal is to reproduce a lowest order calculation in classical field theory. Since we aim for a classical result, you might think that it is obvious that we should only compute tree diagrams: after all, it is standard lore that loops are quantum corrections! However, this is not quite accurate. We will indeed compute a tree diagram, but the justification is that we wish for a lowest order result in classical field theory. Loops are relevant for higher orders in the classical limit when massive particles are present [80].

\subsection{The scattering amplitude}

We have argued that the calculation of interest should be a tree five-point amplitude. This is a very straightforward calculation using Feynman diagrams. There are a total of seven Feynman diagrams, two of which involve four point vertices. Thus there are five cubic diagrams, shown in figure 2, whose corresponding colour factors are

$$
\begin{aligned}
& c_{A}=\left(T_{1}^{a} \cdot T_{1}^{b}\right) T_{2}^{b}, \\
& c_{B}=\left(T_{1}^{b} \cdot T_{1}^{a}\right) T_{2}^{b}, \\
& c_{C}=f^{a b c} T_{1}^{b} T_{2}^{c}, \\
& c_{D}=T_{1}^{b}\left(T_{2}^{a} \cdot T_{2}^{b}\right), \\
& c_{E}=T_{1}^{b}\left(T_{2}^{b} \cdot T_{2}^{a}\right) .
\end{aligned}
$$

\footnotetext{
${ }^{2}$ If any of the representations $R_{i}$, say $R_{1}$ were to be the adjoint, it may be appropriate to include vector states in the double copy built up from one gauge field times one $\Phi_{1}$. However in this case we may choose the representations at will and avoid these unwanted states. We thank Radu Roiban for discussions on this point.
} 


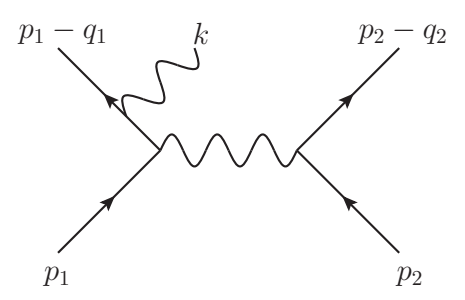

(a) Diagram A

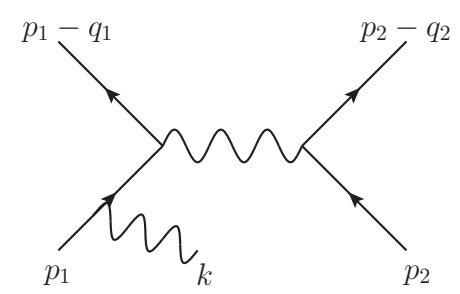

(b) Diagram B

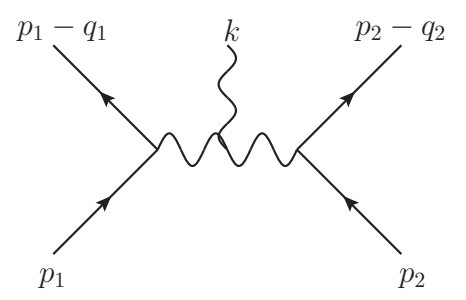

(c) Diagram C

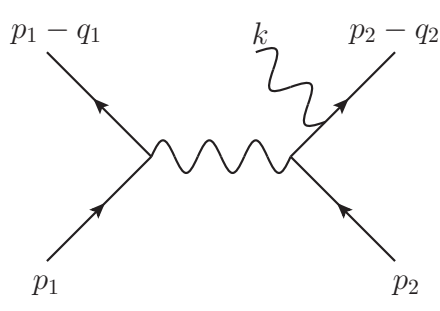

(d) Diagram D

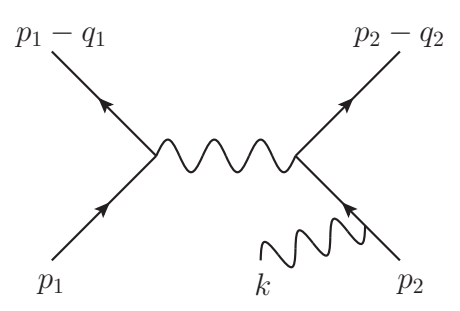

(e) Diagram E

Figure 2. The five cubic diagrams for inelastic scalar scattering with gluon production in gauge theory, where time runs vertically.

The notation $T_{1}^{a} \cdot T_{1}^{b}$ indicates a matrix contraction of the group generators in representation $R_{1} ; c_{A}$ is an element of the tensor product space $R_{1} \otimes R_{2}$.

The total amplitude may then be written as

$$
\mathcal{A}=\frac{n_{A} c_{A}}{d_{A}}+\frac{n_{B} c_{B}}{d_{B}}+\frac{n_{C} c_{C}}{d_{C}}+\frac{n_{D} c_{D}}{d_{D}}+\frac{n_{E} c_{E}}{d_{E}},
$$

where the kinematic numerators $n_{i}$ and the propagators $d_{i}$ can be obtained by computing the seven Feynman diagrams, and assigning terms from the four point vertices to cubic diagrams according to their colour factors. Working in Feynman gauge, ${ }^{3}$ the explicit results for the numerators are:

$$
\begin{aligned}
& n_{A}=\left(2 p_{1}+q_{2}\right) \cdot\left(2 p_{2}-q_{2}\right) \epsilon \cdot\left(2 p_{1}+2 q_{2}\right)-\left(2 p_{1} \cdot q_{2}+q_{2}^{2}\right) \epsilon \cdot\left(2 p_{2}-q_{2}\right), \\
& n_{B}=\left(2 p_{1}-k-q_{1}\right) \cdot\left(2 p_{2}-q_{2}\right) 2 \epsilon \cdot p_{1}+2 p_{1} \cdot k \epsilon \cdot\left(2 p_{2}-q_{2}\right), \\
& n_{C}=\left(2 p_{1}-q_{1}\right)^{\mu}\left(2 p_{2}-q_{2}\right)^{\rho}\left[\left(k+q_{2}\right)_{\mu} \eta_{\nu \rho}+\left(q_{1}-q_{2}\right)_{\nu} \eta_{\rho \mu}-\left(k+q_{1}\right)_{\rho} \eta_{\mu \nu}\right] \epsilon^{\nu}, \\
& n_{D}=\left(2 p_{1}-q_{1}\right) \cdot\left(2 p_{2}+q_{1}\right) \epsilon \cdot\left(2 p_{2}+2 q_{1}\right)-\left(2 p_{2} \cdot q_{1}+q_{1}^{2}\right) \epsilon \cdot\left(2 p_{1}-q_{1}\right), \\
& n_{E}=\left(2 p_{1}-q_{1}\right) \cdot\left(2 p_{2}-k-q_{2}\right) 2 \epsilon \cdot p_{2}+2 p_{2} \cdot k \epsilon \cdot\left(2 p_{1}-q_{1}\right) .
\end{aligned}
$$

Notice that the symmetry of the situation requires that $n_{D}$ is simply equal to $n_{A}$ with particles 1 and 2 interchanged; similarly $n_{E}$ can be obtained from $n_{B}$.

The propagators are straightforward to compute, yielding

$$
\begin{aligned}
& d_{A}=\left(2 p_{1} \cdot q_{2}+q_{2}^{2}\right) q_{2}^{2}, \\
& d_{B}=-2 p_{1} \cdot k q_{2}^{2}, \\
& d_{C}=q_{1}^{2} q_{2}^{2}, \\
& d_{D}=\left(2 p_{2} \cdot q_{1}+q_{1}^{2}\right) q_{1}^{2}, \\
& d_{E}=-2 p_{2} \cdot k q_{1}^{2} .
\end{aligned}
$$

\footnotetext{
${ }^{3}$ As they will not be relevant for our discussion, we omit factors of $i$ and couplings in our Feynman rules.
} 
Once again, symmetry relates $d_{A}$ to $d_{D}$ and $d_{B}$ to $d_{E}$. From now on, we will exploit this symmetry and omit the explicit expressions for diagrams $D$ and $E$.

Before we construct the double copy, we must ensure that our numerators satisfy colour-kinematics duality; if they do not, we will need to modify them appropriately. In the case at hand, the colour factors satisfy precisely two identities:

$$
c_{A}-c_{B}=c_{C} ; \quad c_{D}-c_{E}=c_{C} .
$$

Therefore, we can construct a new gauge invariant amplitude via the double copy

$$
\mathcal{M}=\frac{n_{A} n_{A}}{d_{A}}+\frac{n_{B} n_{B}}{d_{B}}+\frac{n_{C} n_{C}}{d_{C}}+\frac{n_{D} n_{D}}{d_{D}}+\frac{n_{E} n_{E}}{d_{E}},
$$

if the kinematic numerators satisfy

$$
n_{A}-n_{B}=-n_{C} ; \quad n_{E}-n_{D}=-n_{C}
$$

In fact, no modification is necessary for this five-point tree level case: the kinematic identities (3.8) hold immediately in Feynman gauge, as a direct calculation using the list of numerators in equation (3.4) shows [39]. This favourable situation is not expected to hold in general (i.e. for higher point amplitudes).

In view of the success of the double copy, we now have an expression, equation (3.7), for a scattering amplitude in a gravitational theory. The motivation to construct this amplitude was to compare it to classical gravitational scattering: let us now see whether we have been successful.

\subsection{Large mass expansion}

In the previous section, we calculated an amplitude corresponding to the scattering of two scalar particles, accompanied by the emission of gravitational radiation. We would now like to compare this to the classical scattering results in the Goldberger-Ridgway approach of refs. [22, 23], reviewed here in section 2.2, and it is not immediately clear how the two calculations are related. There are two issues to be considered. The first is what constitutes classical scattering. Generally accepted wisdom dictates that tree-level diagrams correspond to classical physics, and loop diagrams provide quantum corrections. However, there are subtleties in this argument, as discussed in detail in ref. [80], whose conclusion is that loop integrals do indeed have a classical component. To see why, one may consider the Lagrangian of our theory with explicit factors of $\hbar$ reinstated:

$$
\mathcal{L}=-\frac{1}{2} \operatorname{tr} F^{\mu \nu} F_{\mu \nu}+\left[\sum_{i}\left(D_{\mu} \Phi_{i}\right)^{\dagger}\left(D^{\mu} \Phi_{i}\right)-\frac{m_{i}^{2}}{\hbar^{2}}|\Phi|^{2}\right],
$$

where $D_{\mu}=\partial_{\mu}+i g A_{\mu} / \hbar$. In any given amplitude, factors of $\hbar$ will occur associated with the couplings and with the masses. Our amplitude is homogeneous in the couplings, but not in the masses, so to take the classical limit we should treat the mass $m_{i}$ as large. This is the source of classical corrections from both tree and loop diagrams [80]. ${ }^{4}$ Here, we are

\footnotetext{
${ }^{4}$ Note that the fact that classical corrections can come from either tree or loop diagrams also follows from the need for infrared singularities to cancel between real and virtual graphs [81-83].
} 
requiring resolvable radiation in the final state, and studying the lowest order Feynman diagrams for this to occur. Thus, we do not need to add loop corrections to reproduce the results of section 2.2 .

The second issue in relating the amplitude and equation of motion approaches is the fact that the latter approach includes an expansion in the deflections of the scattering particles. There are thus two separate expansion parameters in principle: the coupling constant $g$, and the momentum transfer between the scattering particles and the radiated graviton, which measures the degree of deflection. As argued in reference [22], these expansions are correlated, in that the degree of deflection increases with each order of the coupling. In our amplitude calculation, we therefore need to identify the relevant expansion parameter that isolates this behaviour.

In fact, this idea already exists in the literature. In particular, refs. [84, 85] concerned the classification of radiation in both gauge theory and gravity, up to and including "nextto-soft" terms in a systematic expansion in the radiated momentum. The authors developed a physical picture (based on the worldline formalism in quantum field theory [86-88]), in which the propagators for the scattering particles are replaced by quantum mechanical (first-quantised) path integrals. These path integrals can be calculated perturbatively, which corresponds to a sum over the scattering particle trajectories, and thus the possible deflections of the particles. Furthermore, the expansion of each path integral was achieved by rescaling particle momenta according to $p^{\mu} \rightarrow \lambda p^{\mu}$, before expanding in inverse power of $\lambda$. This is precisely the large mass expansion alluded to above.

To extract the large masses, we will express the momenta of the incoming particles in terms of proper velocities, so $p_{i}^{\mu}=m_{i} v_{i}^{\mu}$. Then it is clear that the on-shell requirement for the incoming states translates to the statement that $v_{i}^{2}=1$. But we must also require that the outgoing states are on-shell, so

$$
\begin{aligned}
\left(p_{i}-q_{i}\right)^{2} & =m_{i}^{2}-2 m_{i} v_{i} \cdot q_{i}+q_{i}^{2}=m_{i}^{2} \\
\Rightarrow \quad 2 m_{i} v_{i} \cdot q_{i} & =q_{i}^{2} .
\end{aligned}
$$

This equality is necessary to keep our amplitude on shell, so it is important to respect it scrupulously while performing the large mass expansion. Thus we treat the quantity $v_{i} \cdot q_{i}$ as of order $1 / m$.

It will be useful to introduce some notation to keep track of various terms in the large mass expansion. The dominant terms in the list, equation (3.4), of our numerators is of order $m^{3}$, and there are subleading corrections of order $m^{2}$ and lower. We will indicate this by writing $n_{i}=n_{i}^{(3)}+n_{i}^{(2)}+\cdots$. The dominant terms in the numerators are

$$
\begin{aligned}
& n_{A}^{(3)}=8 m_{1}^{2} m_{2} v_{1} \cdot v_{2} \epsilon \cdot v_{1}, \\
& n_{B}^{(3)}=8 m_{1}^{2} m_{2} v_{1} \cdot v_{2} \epsilon \cdot v_{1}, \\
& n_{C}^{(3)}=0 .
\end{aligned}
$$


We similarly expand the propagators $d_{i}$ in powers of the masses. They become

$$
\begin{aligned}
& d_{A}=\left(2 p_{1} \cdot q_{2}+q_{2}^{2}\right) q_{2}^{2}=2 m_{1} v_{1} \cdot k q_{2}^{2}+O(1 / m), \\
& d_{B}=-2 p_{1} \cdot k q_{2}^{2}=-2 m_{1} v_{1} \cdot k q_{2}^{2}, \\
& d_{C}=q_{1}^{2} q_{2}^{2} .
\end{aligned}
$$

We write these as $d_{i}=d_{i}^{(1)}+d_{i}^{(0)}+\cdots$.

At leading order in large masses, there is a considerable simplification. Since $n_{A}^{(3)}=n_{B}^{(3)}$ and $n_{D}^{(3)}=n_{E}^{(3)}$ to this order, while $d_{A}^{(1)}=-d_{B}^{(1)}$ and $d_{D}^{(1)}=-d_{E}^{(1)}$, it is easy to see that the dominant term in the gravitational amplitude vanishes. We need to go one order deeper in the large mass expansion to find anything interesting.

Straightforward Taylor expansions of the exact numerators in equation (3.4) lead to the next order corrections

$$
\begin{aligned}
& n_{A}^{(2)}=8 m_{1} m_{2} v_{1} \cdot v_{2} \epsilon \cdot q_{2}-4 m_{1} m_{2} v_{1} \cdot q_{2} \epsilon \cdot v_{2}-4 m_{1}^{2} \epsilon \cdot v_{1} v_{1} \cdot q_{2}, \\
& n_{B}^{(2)}=4 m_{1} m_{2}\left(v_{1} \cdot k \epsilon \cdot v_{2}-\epsilon \cdot v_{1} v_{2} \cdot k-\epsilon \cdot v_{1} v_{2} \cdot q_{1}\right)-4 m_{1}^{2} \epsilon \cdot v_{1} v_{1} \cdot q_{2}, \\
& n_{C}^{(2)}=8 m_{1} m_{2}\left(v_{1} \cdot q_{2} \epsilon \cdot v_{2}+q_{1} \cdot \epsilon v_{1} \cdot v_{2}-v_{2} \cdot q_{1} v_{1} \cdot \epsilon\right) .
\end{aligned}
$$

Meanwhile the corrections to the full propagators in equation (3.5) are

$$
\begin{aligned}
& d_{A}^{(0)}=q_{2}^{2}-q_{1}^{2}, \\
& d_{B}^{(0)}=0, \\
& d_{C}^{(0)}=d_{C}=q_{1}^{2} q_{2}^{2} .
\end{aligned}
$$

In terms of these quantities, the full gravitational amplitude is

$$
\mathcal{M}_{\mathrm{cl}}=-\frac{\left(n_{A}^{(3)}\right)^{2}}{\left(d_{A}^{(1)}\right)^{2}} d_{A}^{(0)}+2 \frac{n_{A}^{(3)}\left(n_{A}^{(2)}-n_{B}^{(2)}\right)}{d_{A}^{(1)}}+\frac{\left(n_{C}^{(2)}\right)^{2}}{d_{C}^{(0)}}-\frac{\left(n_{D}^{(3)}\right)^{2}}{\left(d_{D}^{(1)}\right)^{2}} d_{D}^{(0)}+2 \frac{n_{D}^{(3)}\left(n_{D}^{(2)}-n_{E}^{(2)}\right)}{d_{D}^{(1)}} .
$$

Notice that each term has a net four powers of mass. We have also written $\mathcal{M}_{\mathrm{cl}}$ to indicate that this quantity is a classical limit of the tree amplitude. After some algebra, the amplitude can be expressed in terms of the gauge-invariant $P$ and $Q$ vectors defined in equation (2.16). The result is

$$
\begin{aligned}
\mathcal{M}_{\mathrm{cl}}=16 m_{1}^{2} m_{2}^{2} \epsilon_{\mu} \epsilon_{\nu}[ & 4 \frac{P_{12}^{\mu} P_{12}^{\nu}}{q_{1}^{2} q_{2}^{2}}+2 \frac{v_{1} \cdot v_{2}}{q_{1}^{2} q_{2}^{2}}\left(Q_{12}^{\mu} P_{12}^{\nu}+Q_{12}^{\nu} P_{12}^{\mu}\right) \\
& \left.+\left(v_{1} \cdot v_{2}\right)^{2}\left(\frac{Q_{12}^{\mu} Q_{12}^{\nu}}{q_{1}^{2} q_{2}^{2}}-\frac{P_{12}^{\mu} P_{12}^{\nu}}{\left(k \cdot v_{1}\right)^{2}\left(k \cdot v_{2}\right)^{2}}\right)\right] .
\end{aligned}
$$

It is instructive to compare this scattering amplitude against the expression, equation (2.17), for the classical radiation emitted during scattering. Evidently these quantities are closely related: the classical result of equation (2.17) is an integral over the scattering amplitude in the large mass region, times certain factors.

To fully reconcile the classical calculation with the scattering amplitude in the large mass expansion, we need to bear in mind that, classically, the particles are associated with 


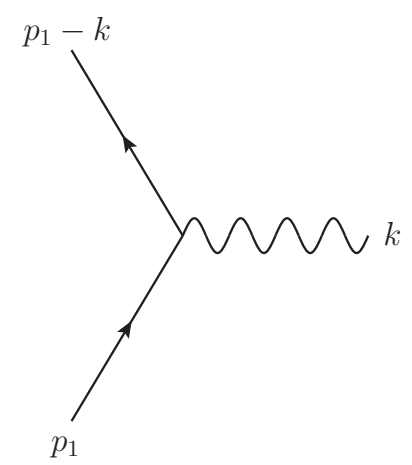

Figure 3. The three point interaction involving the massive gauge particle.

given states in position-space. We can write these as superpositions of momentum-space states as follows:

$$
\left|\psi_{i}\right\rangle=\int đ q_{i} \delta\left(v_{i} \cdot q_{i}\right) e^{i q_{i} \cdot\left(b_{i}-x\right)}\left|q_{i}\right\rangle
$$

where $\left|q_{i}\right\rangle$ is a momentum eigenstate of the scalar field $\Phi_{i}$. Working in the rest-frame of this particle, where $v_{i}=(1,0,0,0)$, we can write the state as

$$
\left|\psi_{i}\right\rangle=\int \frac{d^{3} q_{i}}{(2 \pi)^{3}} e^{-i \underline{q_{i}} \cdot\left(\underline{b_{i}}-\underline{x}\right)}\left|q_{i}\right\rangle
$$

which can be recognised as simply the wavefunction of a particle localised at the threedimensional position $\underline{x}=\underline{b_{i}}$.

\section{Removing the dilaton}

The theory obtained via the double copy of pure Yang-Mills theory is gravity, coupled to a dilaton and an antisymmetric tensor, the axion. So far, we have been investigating a slightly different case: the double copy of Yang-Mills theory coupled to a massive scalar. In the double copy, we will therefore obtain gravity; a massless scalar dilaton; a massless axion; and a massive scalar particle. The graviton, dilaton and axion combine to make a single product graviton $H_{\mu \nu}$, and we must remove the scalar degrees of freedom in order to obtain pure General Relativity. Given that all of our solutions for $H_{\mu \nu}$ will be manifestly symmetric in the indices $\mu$ and $\nu$, the axion never appears in what follows. However, we must still construct a procedure for removing the dilaton. We begin by investigating how the dilaton couples to the massive scalar particles in the scattering process.

\subsection{Double copy and massive amplitudes}

It is straightforward to construct three-point scattering amplitudes involving the massive particle. In the gauge theory, there is only the three point amplitude shown in figure 3, corresponding to a gauge field interacting with the scalar current.

The amplitude corresponding to this diagram is simply

$$
\mathcal{A}=2 p_{1} \cdot \epsilon T_{1},
$$


where $\epsilon$ is the polarisation vector associated with the vector particle. Notice that the requirement that all three external states are on-shell requires that $p_{1} \cdot k=0$.

Now we pass to the double-copied theory; we choose the polarisation vector in the second copy to be $\tilde{\epsilon}$. The amplitude is

$$
\mathcal{M}=4 p_{1} \cdot \epsilon p_{1} \cdot \tilde{\epsilon}
$$

To determine how the massive particle couples to the dilaton, axion and graviton, we decompose the outer product of polarisation vectors into irreducible representations of the little group associated with the massless momentum $k$,

$$
\epsilon^{\mu} \tilde{\epsilon}^{\nu}=\underbrace{\frac{1}{2}\left(\epsilon^{\mu} \tilde{\epsilon}^{\nu}+\epsilon^{\nu} \tilde{\epsilon}^{\mu}-\frac{2}{d-2} \epsilon \cdot \tilde{\epsilon} \eta_{\mathrm{lg}}^{\mu \nu}\right)}_{\text {graviton }}+\underbrace{\frac{1}{2}\left(\epsilon^{\mu} \tilde{\epsilon}^{\nu}-\epsilon^{\nu} \tilde{\epsilon}^{\mu}\right)}_{\text {axion }}+\underbrace{\left(\frac{\epsilon \cdot \tilde{\epsilon}}{d-2} \eta_{\mathrm{lg}}^{\mu \nu}\right)}_{\text {dilaton }},
$$

where

$$
\eta_{\mathrm{lg}}^{\mu \nu}=\eta_{\mu \nu}-\frac{k_{\mu} q_{\nu}+k_{\nu} q_{\mu}}{k \cdot q}
$$

and $q$ is a choice of gauge satisfying $q \cdot q=0$. The symmetry of $\mathcal{M}$ under interchanging $\epsilon$ and $\tilde{\epsilon}$ projects the antisymmetric tensor, corresponding to the axion, out. Meanwhile, the graviton and dilaton components of $\mathcal{M}$ are

$$
\begin{aligned}
\mathcal{M}_{\text {graviton }} & =4 p_{1}^{\mu} p_{1}^{\nu} e_{\mu \nu} \\
\mathcal{M}_{\text {dilaton }} & =\frac{4}{d-2} m_{1}^{2}
\end{aligned}
$$

where $e_{\mu \nu}$ is the traceless, symmetric polarisation tensor of the graviton.

A key point is that in the massive case $m_{1} \neq 0$, there is a coupling between the massless dilaton and the massive scalar field. Correspondingly, a dilaton propagates in intermediate states in the five point amplitude we discussed previously in section 3. Classically, the massive particles interact through a massless scalar force in addition to the gravitational force. While this is a natural feature of the double copy, it is desirable to be able to turn off this coupling to the dilaton: indeed, any application of the double copy to physical black hole scattering requires some means of disentangling contributions from dilatons. So let us now face this issue: how can we simply remove the dilaton diagrams from the double copy process?

\subsection{Dilatons in diagrams}

It is generally straightforward to identify the contributions of particular substates in scattering amplitudes by looking at their cuts or factorisation channels. In the present case, we already know how the dilaton appears in the five point amplitude. It is convenient to consider two categories of diagram involving the dilaton: those with external dilatons, for example the diagrams in figure 4, and those without any external dilatons. Examples of diagrams in this second class are shown in figure 5. Of course dilatons may be present as virtual states in both categories. 

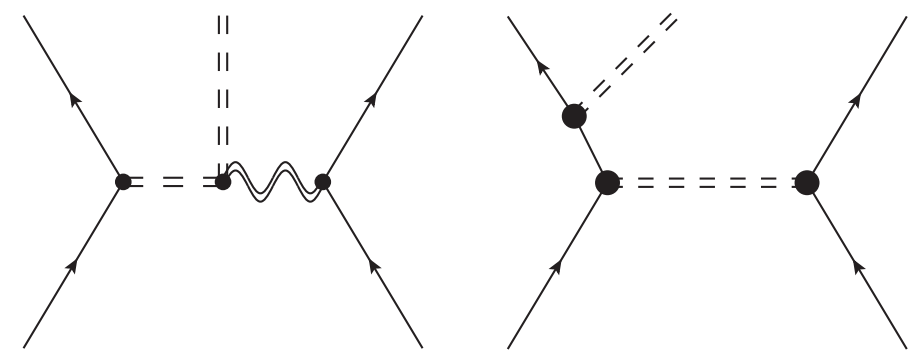

Figure 4. Diagrams with dilatons radiated into the final state. The dilaton is represented by the double dashed line, while the graviton is the double wavy line.
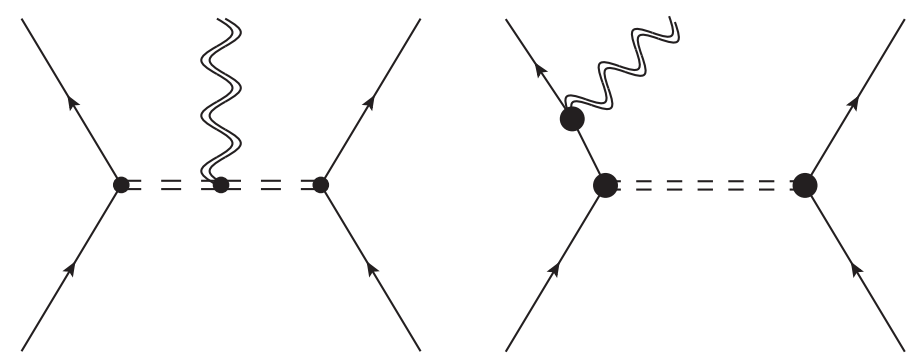

Figure 5. Types of diagram with only intermediate, virtual, dilaton states.

The first class of diagram, with dilatons in the final state, is trivial to deal with. We simply project our amplitude onto the traceless symmetric component by hand by replacing $\epsilon^{\mu} \tilde{\epsilon}^{\nu} \rightarrow e^{\mu \nu}$. In doing so, we have thrown away the dilaton polarisation tensor which is the trace term present in the tensor decomposition of the outer product of polarisation vectors, equation (4.3). This replacement explicitly forces the external state to be a graviton, rather than a dilaton - thus removing all diagrams in our first category. But this procedure does not remove the diagrams in our second category, in which virtual dilatons may be present.

A few methods for removing virtual dilatons suggest themselves. The first method, advocated in [21], is to insert a projector onto graviton states at vertices in which the massive scalar line may interact with a dilaton. A second, related, option is to insert a projector on the intermediate lines. But there is also a third option which is very simple to implement. We observe that the dilaton is a scalar particle propagating in the double copy. It is possible to remove such scalar particles by introducing a ghost: another scalar particle in the double copy, but where the double copy is defined by introducing a negative sign any time this particle appears. This method has been used in the context of the double copy by Johansson and Ochirov [38] to obtain pure gravity as a double copy.

We will therefore introduce a new massless scalar state in our gauge theory. We can constrain how the new scalar behaves by requiring that it removes the coupling between our massive scalar and the dilaton in the double copy. In particular, the ghost must remove the diagrams in figure 5. We will therefore assume that the ghost couples to two massive scalars, and that the ghost is charged under the gauge symmetry, transforming in the 


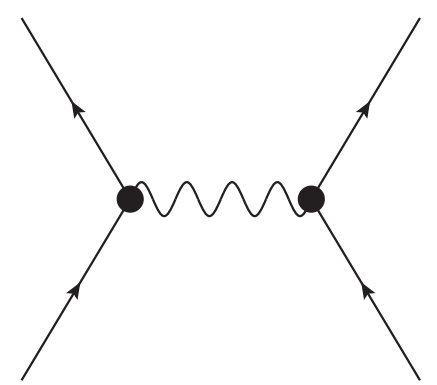

(a) Gauge boson mediator

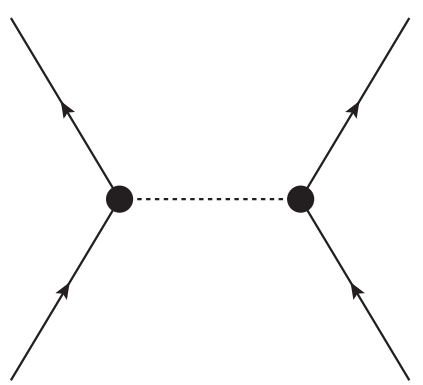

(b) Ghost mediator

Figure 6. Four point scattering in the extended gauge theory of equation (4.7).

adjoint representation. The Lagrangian of the theory is

$$
\mathcal{L}=-\frac{1}{2} \operatorname{tr} F_{\mu \nu} F^{\mu \nu}+\operatorname{tr} D^{\mu} \chi D_{\mu} \chi+\sum_{i}\left[\left(D_{\mu} \Phi_{i}\right)^{\dagger} D^{\mu} \Phi_{i}-m_{i}^{2} \Phi_{i}^{\dagger} \Phi_{i}-2 X m_{i} \Phi_{i}^{\dagger} \chi \Phi_{i}\right],
$$

where $\chi$ is the adjoint ghost, and $X$ is a coupling to be determined. ${ }^{5}$

\subsection{Example at four points}

To see how the procedure works in the simplest case, we turn to elastic two-particle scattering. We will determine the amplitude for two massive scalar particles scattering off one another in General Relativity from the double copy. Beginning in gauge theory, there are only two diagrams to compute, as shown in figure 6 .

We denote the incoming momenta by $p_{1}$ and $p_{2}$ and let the momentum transfer be $q$. As we will treat the gauge and ghost mediator cases slightly differently, we present the contributions separately; they are

$$
\begin{aligned}
& \mathcal{A}_{\text {gauge }}=\frac{4 p_{1} \cdot p_{2}+q^{2}}{q^{2}} T_{1} \cdot T_{2}, \\
& \mathcal{A}_{\text {ghost }}=X^{2} \frac{4 m_{1} m_{2}}{q^{2}} T_{1} \cdot T_{2} .
\end{aligned}
$$

At this order, colour-kinematics duality is trivial so the double copy is immediate. We define the double copy for the ghost by inserting a sign, so that the gravitational amplitude is

$$
\mathcal{M}=\frac{1}{q^{2}}\left[\left(4 p_{1} \cdot p_{2}+q^{2}\right)^{2}-X^{4}\left(4 m_{1} m_{2}\right)^{2}\right] .
$$

Now, on the factorisation channel where $q^{2}=0$, the quantity $q^{2} \mathcal{M}$ must factorize into a product of three point amplitudes, summed over intermediate helicities. We find

$$
\begin{aligned}
q^{2} \mathcal{M} & \underset{q^{2} \rightarrow 0}{\longrightarrow}\left(4 p_{1} \cdot p_{2}\right)^{2}-X^{4}\left(4 m_{1} m_{2}\right)^{2} \\
& =8 p_{1}^{\mu} p_{1}^{\mu^{\prime}} p_{2}^{\nu} p_{2}^{\nu^{\prime}}\left[\eta_{\mu \nu} \eta_{\mu^{\prime} \nu^{\prime}}+\eta_{\mu \nu^{\prime}} \eta_{\mu^{\prime} \nu}-2 X^{4} \eta_{\mu \mu^{\prime}} \eta_{\nu \nu^{\prime}}\right] .
\end{aligned}
$$

\footnotetext{
${ }^{5}$ The factor of $m_{i}$ in the coupling is inserted so that $X$ is dimensionless, and using the knowledge from equation (4.6) that the dilaton couples to mass.
} 
In General Relativity, the quantity in square brackets in the last line must be equal to the de Donder projector

$$
P_{\mu \mu^{\prime} \nu \nu^{\prime}}=\left[\eta_{\mu \nu} \eta_{\mu^{\prime} \nu^{\prime}}+\eta_{\mu \nu^{\prime}} \eta_{\mu^{\prime} \nu}-\frac{2}{d-2} \eta_{\mu \mu^{\prime}} \eta_{\nu \nu^{\prime}}\right]
$$

up to pure gauge terms. We therefore conclude that

$$
X^{4}=\frac{1}{d-2} .
$$

\subsection{Inelastic scattering}

Our next task is to re-compute the five-point scattering amplitude in gauge theory, including the new state. It is convenient to use the same cubic topologies as presented in figure 2. The contributions to the amplitude due to the presence of the $\chi$ are given by

$$
\mathcal{A}_{\text {ghost }}=\frac{c_{A} n_{A}^{\prime}}{d_{A}}+\frac{c_{B} n_{B}^{\prime}}{d_{B}}+\frac{c_{C} n_{C}^{\prime}}{d_{C}}+\frac{c_{D} n_{D}^{\prime}}{d_{D}}+\frac{c_{E} n_{E}^{\prime}}{d_{E}} .
$$

A straightforward calculation yields the new terms in the numerators $n_{A}^{\prime}, n_{B}^{\prime}$ and $n_{C}^{\prime}$. These new terms are

$$
\begin{aligned}
& n_{A}^{\prime}=4 m_{1} m_{2} X^{2} 2 \epsilon \cdot\left(p_{1}+q_{2}\right), \\
& n_{B}^{\prime}=4 m_{1} m_{2} X^{2} 2 \epsilon \cdot p_{1}, \\
& n_{C}^{\prime}=-4 m_{1} m_{2} X^{2} \epsilon \cdot\left(q_{1}-q_{2}\right) .
\end{aligned}
$$

The numerators to $n_{D}^{\prime}$ and $n_{E}^{\prime}$ can be obtained by swapping the particle labels 1 and 2 in $n_{A}^{\prime}$ and $n_{B}^{\prime}$ as before. It is easy to see that the these numerators satisfy the relation

$$
n_{A}^{\prime}-n_{B}^{\prime}=n_{C}^{\prime}
$$

so that colour-kinematics duality is satisfied, and we can construct the double copy as before. The contribution of the new state to the amplitude is

$$
\mathcal{M}_{\text {scalar }}=\frac{n_{A}^{\prime} n_{A}^{\prime}}{d_{A}}+\frac{n_{B}^{\prime} n_{B}^{\prime}}{d_{B}}+\frac{n_{C}^{\prime} n_{C}^{\prime}}{d_{C}}+\frac{n_{D}^{\prime} n_{D}^{\prime}}{d_{D}}+\frac{n_{E}^{\prime} n_{E}^{\prime}}{d_{E}}
$$

Inserting the explicit expressions, and replacing the product of polarisation vectors $\epsilon_{\mu} \epsilon_{\nu}$ by the traceless symmetric graviton polarisation tensor $e_{\mu \nu}$ to remove final state dilatons, we find the explicit amplitude

$$
\mathcal{M}_{\text {scalar, }, \mathrm{cl}}=16 m_{1}^{2} m_{2}^{2} e_{\mu \nu}\left[\frac{1}{d-2}\left(\frac{Q_{12}^{\mu} Q_{12}^{\nu}}{q_{1}^{2} q_{2}^{2}}-\frac{P_{12}^{\mu} P_{12}^{\nu}}{\left(k \cdot v_{1}\right)^{2}\left(k \cdot v_{2}\right)^{2}}\right)\right],
$$

where we used the value of $X$, equation (4.14), determined at four points, and we have performed a large mass expansion. The Einstein gravity amplitude is then easily obtained by subtracting the scalar contributions from the gravitational amplitude computed in the previous section. This is

$$
\mathcal{M}_{\mathrm{GR}}=\mathcal{M}_{\mathrm{cl}}-\mathcal{M}_{\mathrm{scalar}, \mathrm{cl}}
$$


where $\mathcal{M}_{\mathrm{cl}}$ is defined as in equation (3.17). The Einstein gravity amplitude takes the explicit form

$$
\begin{aligned}
\mathcal{M}_{\mathrm{GR}}=16 m_{1}^{2} m_{2}^{2} e_{\mu \nu}[ & 4 \frac{P_{12}^{\mu} P_{12}^{\nu}}{q_{1}^{2} q_{2}^{2}}+2 \frac{v_{1} \cdot v_{2}}{q_{1}^{2} q_{2}^{2}}\left(Q_{12}^{\mu} P_{12}^{\nu}+Q_{12}^{\nu} P_{12}^{\mu}\right) \\
& \left.+\left(\left(v_{1} \cdot v_{2}\right)^{2}-\frac{1}{d-2}\right)\left(\frac{Q_{12}^{\mu} Q_{12}^{\nu}}{q_{1}^{2} q_{2}^{2}}-\frac{P_{12}^{\mu} P_{12}^{\nu}}{\left(k \cdot v_{1}\right)^{2}\left(k \cdot v_{2}\right)^{2}}\right)\right] .
\end{aligned}
$$

The classical graviton associated with this amplitude can therefore be taken to be

$$
\begin{aligned}
k^{2} h^{(1) \mu \nu}(k)= & -\frac{m_{1} m_{2}}{8 m_{\mathrm{pl}}^{3(d-2) / 2}} \int đ q_{1} đ q_{2} \delta\left(k-q_{1}-q_{2}\right) \delta\left(q_{1} \cdot v_{1}\right) e^{i q_{1} \cdot b_{1}} \delta\left(q_{2} \cdot v_{2}\right) e^{i q_{2} \cdot b_{2}} \times \\
& {\left[\frac{P_{12}^{\mu} P_{12}^{\nu}}{q_{1}^{2} q_{2}^{2}}+\frac{v_{1} \cdot v_{2}}{2 q_{1}^{2} q_{2}^{2}}\left(Q_{12}^{\mu} P_{12}^{\nu}+Q_{12}^{\nu} P_{12}^{\mu}\right)+\frac{1}{4}\left(\left(v_{1} \cdot v_{2}\right)^{2}-\frac{1}{d-2}\right) \times\right.} \\
& \left.\left(\frac{Q_{12}^{\mu} Q_{12}^{\nu}}{q_{1}^{2} q_{2}^{2}}-\frac{P_{12}^{\mu} P_{12}^{\nu}}{\left(k \cdot v_{1}\right)^{2}\left(k \cdot v_{2}\right)^{2}}\right)\right]
\end{aligned}
$$

up to pure gauge terms. We have checked that this result is accurate by comparison with a far more complicated direct computation in General Relativity coupled to point particles.

\subsection{Relation to classical field computation}

Given that our treatment was motivated by the computation performed by Goldberger and Ridgway, it is fair to ask if the method we employed to remove the dilaton can also be implemented in their framework. We will consider an analogue of the classical gauge theory equations (2.6) for a ghost field $\chi$ that couples to the classical coloured point particles as well as the gluons. The equations defining the contributions from the ghost to the deflection of the colour charged point particles are

$$
\begin{aligned}
\partial^{2} \chi^{a} & =\sum_{i} 2 X g m_{i} \int d \tau c_{i}^{a}(\tau) \delta^{(d)}\left(x-x_{i}(\tau)\right), \\
m_{i} \frac{d^{2} x_{i}^{\mu}(\tau)}{d \tau^{2}} & =2 X g m_{i} \partial^{\mu} \chi^{a}\left(x_{i}(\tau)\right) c_{i}^{a}(\tau)+\cdots \\
\frac{d c_{i}^{a}(\tau)}{d \tau} & =2 X g m_{i} f^{a b c} \chi^{b}\left(x_{i}(\tau)\right) c_{i}^{c}(\tau)+\cdots
\end{aligned}
$$

where the dots indicate deflections in position and colour due to gravity. We can perform now an analogous computation to the one used to get equation (2.12). This will yield the contributions to the gluon field due to the presence of the ghost field $\chi$, and it takes the explicit form

$$
\begin{aligned}
\left.k^{2} A^{(1) a \mu}(k)\right|_{\text {ghost }}= & g^{3} \int \frac{d q_{1} đ q_{2}}{q_{2}^{2}} \delta\left(k-q_{1}-q_{2}\right) \delta\left(q_{1} \cdot v_{1}\right) e^{i q_{1} \cdot b_{1}} \delta\left(q_{2} \cdot v_{2}\right) e^{i q_{2} \cdot b_{2}} \\
& \times 2 m_{1} 2 m_{2} X^{2} \frac{1}{k \cdot p_{1}}\left[i f^{a b c} c_{2}^{(0) b} c_{1}^{(0) c} p_{1}^{\mu}+c_{1}^{(0) a} c_{1}^{(0)} \cdot c_{2}^{(0)}\left(q_{2}^{\mu}+\frac{p_{1}^{\mu}}{k \cdot p_{1}} k \cdot q_{2}\right)\right. \\
& \left.-i f^{a b c} c_{2}^{(0) b} c_{1}^{(0) c} \frac{\left(q_{1}^{\mu}-q_{2}^{\mu}\right)}{q_{1}^{2}} k \cdot p_{1}\right]+(1 \leftrightarrow 2) .
\end{aligned}
$$


If we consider the leading terms in a large mass expansion of the ghost contributions to the scattering amplitude in equation (4.15):

$$
\begin{aligned}
& \frac{c_{A} n_{A}^{\prime}}{d_{A}} \rightarrow 4 m_{1} m_{2} X^{2} c_{A}\left(\frac{\epsilon \cdot p_{1}}{p_{1} \cdot k}+\frac{\epsilon \cdot q_{2}}{p_{1} \cdot k}+\frac{\epsilon \cdot p_{1} q_{2} \cdot k}{\left(p_{1} \cdot k\right)^{2}}\right) \\
& \frac{c_{B} n_{B}^{\prime}}{d_{B}} \rightarrow-4 m_{1} m_{2} X^{2} c_{B} \frac{\epsilon \cdot p_{1}}{p_{1} \cdot k} \\
& \frac{c_{C} n_{C}^{\prime}}{d_{C}} \rightarrow-4 m_{1} m_{2} X^{2} c_{C} \frac{\epsilon \cdot\left(q_{1}-q_{2}\right)}{q_{1}^{2} q_{2}^{2}}
\end{aligned}
$$

it is easy to see that the equations (4.25) and (4.26) combine to yield the first terms of equation (4.24), while the contributions from equation (4.27) are directly responsible for the final line of equation (4.24). Thus we see that we can indeed remove the dilaton pollution, this time completely within the framework of classical perturbation theory.

This result shows a direct link between a classical computation similar to that of refs. [22, 23] and an amplitude, so we may ask if the double copy (scalar) scattering amplitude from equation (4.19) can be obtained using a set of replacements similar to those proposed in [22]:

$$
\begin{aligned}
c_{i}^{(0) a} & \rightarrow m_{i} v_{i}^{\mu}, \\
i f^{a b c} & \rightarrow \frac{1}{2} \Gamma^{\mu \nu \rho}\left(q_{1}, q_{2}, q_{3}\right) .
\end{aligned}
$$

It is not difficult to see that such double copy rules will not land on the amplitude from equation (4.19). Instead, it is the set of replacements

$$
\begin{aligned}
c_{1}^{(0) a} c_{1}^{(0)} \cdot c_{2}^{(0)} & \rightarrow 2 m_{1} 2 m_{2} X^{2} p_{1}^{\mu}, \\
i f^{a b c} c_{2}^{(0) b} c_{1}^{(0) c} & \rightarrow 2 m_{1} 2 m_{2} X^{2} \frac{1}{2}\left(q_{1}^{\mu}-q_{2}^{\mu}\right),
\end{aligned}
$$

that one needs to obtain the desired amplitude. Of course, this is because the process we are considering now depends on the dynamics of the ghost field, and so it should be no surprise that the replacement in equation (4.31) involves the ghost-gluon vertex instead of the three gluon vertex of equation (4.29). Nevertheless, this highlights one advantage of the scattering amplitudes: when they are available, it is straightforward to take the double copy. But in situations where scattering amplitudes are not available, it could well be that developing these replacement rules, and supplementing them by some notion of colourkinematics duality, could allow the double copy to access entirely new physical regimes.

\section{Discussion and conclusions}

We hope this work represents a step towards using the double copy as a tool for understanding the classical physics of General Relativity. Future gravitational wave observatories, such as LISA or the Einstein telescope, will operate at higher precision, so the demand for understanding the finest details of gravity will become more pressing. Since precision has always been a driving force in the study of scattering amplitudes, it is natural to investigate whether amplitudes methods have relevance for precision General Relativity. 
We began by reformulating the leading perturbative term in the inelastic gravitational scattering of two singularities as a tree scattering amplitude, following Goldberger and Ridgway's double-copy based calculation. This reformulation allowed us to analyze the factorisation structure of the calculation, and to identify one method of removing the dilaton field which was present in the original calculation. We did so by introducing a new scalar field in the gauge theory which is double-copied to gravity. This scalar is treated as a ghost, in a manner similar to Johansson and Ochirov's construction [38] of loop amplitudes in pure gravity. However, we have not shown that our method will work at higher orders. Indeed it is likely that there will be issues, since ghosts built from double copies of scalars (rather than double copies of spinors) encounter problems at two loops [38]. Our attitude is that an understanding of how best to remove the dilaton will depend on how one computes higher order corrections to the classical scattering process, which we leave for future work.

An interesting feature of Goldberger and Ridgway's work was an unusual implementation of the double copy, equation (2.13). Essentially, they replaced the colour structure constants by the kinematic Yang-Mills three point vertex, and the colour factors by the momenta. This is a little puzzling, because one would expect that some work should be done to synchronise the colour and kinematic structure in the calculation. In our amplitudebased approach, the standard BCJ replacement of colour factors by kinematic numerators was available. But we also encountered a simplification: colour-kinematics duality holds for free (in Feynman gauge.) This simplification is most unlikely to pertain to higher orders, so we anticipate that the replacements Goldberger and Ridgway performed will at least need to be supplemented by some kind of colour-kinematics duality condition in more general cases. On a related note, it was satisfying to see a version of the Goldberger-Ridgway replacement appear in the context of our scalar double copy, equation (4.31). It could well be that in situations where scattering amplitudes are unavailable, a version of the double copy based on these kinds of replacement rules could still work. This may allow the double copy to be used in entirely new ways.

We believe that there is considerable scope for further work on applying the double copy in the context of classical General Relativity. There is obvious motivation to pursue this work to higher orders in perturbation theory, in the cases of elastic and inelastic scattering. It will also be important to determine how to handle the angular momentum of a black hole. In this work, we were insensitive to black hole spin, but higher order corrections will probe this aspect. One issue that is likely to arise is another kind of unwanted state propagating in the double copy: the axion. The axion cannot couple to a spinless point particle, but when a non-trivial spin vector is present a coupling exists. Therefore it is likely to be necessary to remove propagating axions; this is an issue which has already been addressed in loop calculations [38]. This could be an additional complication, but nevertheless it is worth noticing recent, encouraging, progress in capturing spin effects in classical GR [10, 89].

Finally, our goal has been to develop the double copy as a tool to simplify perturbation theory in General Relativity. But the double copy is not the only new idea for simplifying GR. It could be that a better approach [90-92] is to design a new Lagrangian for GR, equivalent to the Einstein-Hilbert Lagrangian, but exhibiting a simpler perturbative expansion. 
This may lead to a simpler algorithm for determining precision gravitational effects. Such a procedure still has the double copy at its heart, however, and thus we expect the double copy to play a key role in gravitational perturbation theory in the coming years.

\section{Acknowledgments}

We thank Zvi Bern, Marco Chiodaroli, Walter Goldberger, Enrico Herrmann, David Kosower, Ricardo Monteiro, Alexander Ochirov, Alec Ridgway, Julio Parra-Martinez and Radu Roiban for helpful discussions. AL is funded by a Conacyt studentship, and thanks the Higgs Centre for hospitality. DOC is an IPPP associate, and thanks the IPPP for on-going support as well as for hospitality during this work. He is supported in part by the Marie Curie FP7 grant 631370 and by the STFC consolidated grant "Particle Physics at the Higgs Centre". IN is supported by STFC studentship ST/N504051/1. Meanwhile, CDW is supported by the U.K. Science and Technology Facilities Council (STFC). This research was supported in part by the National Science Foundation under Grant No. NSF PHY-1125915.

Open Access. This article is distributed under the terms of the Creative Commons Attribution License (CC-BY 4.0), which permits any use, distribution and reproduction in any medium, provided the original author(s) and source are credited.

\section{References}

[1] H. Kawai, D.C. Lewellen and S.H.H. Tye, A Relation Between Tree Amplitudes of Closed and Open Strings, Nucl. Phys. B 269 (1986) 1 [INSPIRE].

[2] Z. Bern, J.J.M. Carrasco and H. Johansson, New Relations for Gauge-Theory Amplitudes, Phys. Rev. D 78 (2008) 085011 [arXiv: 0805.3993] [INSPIRE].

[3] Z. Bern, J.J.M. Carrasco and H. Johansson, Perturbative Quantum Gravity as a Double Copy of Gauge Theory, Phys. Rev. Lett. 105 (2010) 061602 [arXiv: 1004.0476] [INSPIRE].

[4] D. Neill and I.Z. Rothstein, Classical Space-Times from the S Matrix, Nucl. Phys. B 877 (2013) 177 [arXiv:1304.7263] [INSPIRE].

[5] N.E.J. Bjerrum-Bohr, J.F. Donoghue and P. Vanhove, On-shell Techniques and Universal Results in Quantum Gravity, JHEP 02 (2014) 111 [arXiv: 1309.0804] [InSPIRE].

[6] N.E.J. Bjerrum-Bohr, B.R. Holstein, L. Planté and P. Vanhove, Graviton-Photon Scattering, Phys. Rev. D 91 (2015) 064008 [arXiv:1410.4148] [INSPIRE].

[7] N.E.J. Bjerrum-Bohr, J.F. Donoghue, B.R. Holstein, L. Planté and P. Vanhove, Bending of Light in Quantum Gravity, Phys. Rev. Lett. 114 (2015) 061301 [arXiv:1410.7590] [INSPIRE].

[8] N.E.J. Bjerrum-Bohr, J.F. Donoghue, B.R. Holstein, L. Planté and P. Vanhove, Light-like Scattering in Quantum Gravity, JHEP 11 (2016) 117 [arXiv:1609.07477] [INSPIRE].

[9] F. Cachazo and A. Guevara, Leading Singularities and Classical Gravitational Scattering, arXiv:1705.10262 [INSPIRE].

[10] A. Guevara, Holomorphic Classical Limit for Spin Effects in Gravitational and Electromagnetic Scattering, arXiv:1706.02314 [INSPIRE]. 
[11] N.E.J. Bjerrum-Bohr, B.R. Holstein, J.F. Donoghue, L. Planté and P. Vanhove, Illuminating Light Bending, PoS(CORFU2016) 077 [arXiv: 1704.01624] [INSPIRE].

[12] T. Damour, High-energy gravitational scattering and the general relativistic two-body problem, arXiv:1710.10599 [INSPIRE].

[13] R. Monteiro, D. O'Connell and C.D. White, Black holes and the double copy, JHEP 12 (2014) 056 [arXiv: 1410.0239] [INSPIRE].

[14] A. Luna, R. Monteiro, D. O'Connell and C.D. White, The classical double copy for Taub-NUT spacetime, Phys. Lett. B 750 (2015) 272 [arXiv:1507.01869] [INSPIRE].

[15] A.K. Ridgway and M.B. Wise, Static Spherically Symmetric Kerr-Schild Metrics and Implications for the Classical Double Copy, Phys. Rev. D 94 (2016) 044023 [arXiv: 1512.02243] [INSPIRE].

[16] A. Luna, R. Monteiro, I. Nicholson, D. O'Connell and C.D. White, The double copy: Bremsstrahlung and accelerating black holes, JHEP 06 (2016) 023 [arXiv:1603.05737] [INSPIRE].

[17] T. Adamo, E. Casali, L. Mason and S. Nekovar, Scattering on plane waves and the double copy, Class. Quant. Grav. 35 (2018) 015004 [arXiv: 1706. 08925] [INSPIRE].

[18] T. Adamo, E. Casali, L. Mason and S. Nekovar, Amplitudes on plane waves from ambitwistor strings, JHEP 11 (2017) 160 [arXiv:1708.09249] [INSPIRE].

[19] N. Bahjat-Abbas, A. Luna and C.D. White, The Kerr-Schild double copy in curved spacetime, JHEP 12 (2017) 004 [arXiv:1710.01953] [INSPIRE].

[20] M. Carrillo-Gonzalez, R. Penco and M. Trodden, The classical double copy in maximally symmetric spacetimes, arXiv:1711.01296 [INSPIRE].

[21] A. Luna et al., Perturbative spacetimes from Yang-Mills theory, JHEP 04 (2017) 069 [arXiv: 1611.07508] [INSPIRE].

[22] W.D. Goldberger and A.K. Ridgway, Radiation and the classical double copy for color charges, Phys. Rev. D 95 (2017) 125010 [arXiv:1611.03493] [INSPIRE].

[23] W.D. Goldberger, S.G. Prabhu and J.O. Thompson, Classical gluon and graviton radiation from the bi-adjoint scalar double copy, Phys. Rev. D 96 (2017) 065009 [arXiv:1705.09263] [INSPIRE].

[24] A.I. Janis, E.T. Newman and J. Winicour, Reality of the Schwarzschild Singularity, Phys. Rev. Lett. 20 (1968) 878 [INSPIRE].

[25] Z. Bern, T. Dennen, Y.-t. Huang and M. Kiermaier, Gravity as the Square of Gauge Theory, Phys. Rev. D 82 (2010) 065003 [arXiv:1004.0693] [INSPIRE].

[26] Y.-J. Du, B. Feng and C.-H. Fu, BCJ Relation of Color Scalar Theory and KLT Relation of Gauge Theory, JHEP 08 (2011) 129 [arXiv:1105.3503] [INSPIRE].

[27] N.E.J. Bjerrum-Bohr, P.H. Damgaard, R. Monteiro and D. O'Connell, Algebras for Amplitudes, JHEP 06 (2012) 061 [arXiv:1203.0944] [inSPIRE].

[28] C.-H. Fu, Y.-J. Du and B. Feng, An algebraic approach to BCJ numerators, JHEP 03 (2013) 050 [arXiv: 1212.6168] [INSPIRE].

[29] N.E.J. Bjerrum-Bohr, T. Dennen, R. Monteiro and D. O'Connell, Integrand Oxidation and One-Loop Colour-Dual Numerators in N=4 Gauge Theory, JHEP 07 (2013) 092 [arXiv: 1303.2913] [INSPIRE]. 
[30] Y.-J. Du, B. Feng and C.-H. Fu, The Construction of Dual-trace Factor in Yang-Mills Theory, JHEP 07 (2013) 057 [arXiv: 1304.2978] [INSPIRE].

[31] M. Tolotti and S. Weinzierl, Construction of an effective Yang-Mills Lagrangian with manifest BCJ duality, JHEP 07 (2013) 111 [arXiv:1306.2975] [INSPIRE].

[32] J. Nohle, Color-Kinematics Duality in One-Loop Four-Gluon Amplitudes with Matter, Phys. Rev. D 90 (2014) 025020 [arXiv:1309.7416] [INSPIRE].

[33] R. Monteiro and D. O'Connell, The Kinematic Algebras from the Scattering Equations, JHEP 03 (2014) 110 [arXiv:1311.1151] [INSPIRE].

[34] C.-H. Fu, Y.-J. Du and B. Feng, Note on symmetric BCJ numerator, JHEP 08 (2014) 098 [arXiv: 1403.6262] [INSPIRE].

[35] P. Mastrolia, A. Primo, U. Schubert and W.J. Torres Bobadilla, Off-shell currents and color-kinematics duality, Phys. Lett. B 753 (2016) 242 [arXiv: 1507.07532] [INSPIRE].

[36] C.-H. Fu and K. Krasnov, Colour-Kinematics duality and the Drinfeld double of the Lie algebra of diffeomorphisms, JHEP 01 (2017) 075 [arXiv:1603.02033] [INSPIRE].

[37] R.W. Brown and S.G. Naculich, BCJ relations from a new symmetry of gauge-theory amplitudes, JHEP 10 (2016) 130 [arXiv:1608.04387] [INSPIRE].

[38] H. Johansson and A. Ochirov, Pure Gravities via Color-Kinematics Duality for Fundamental Matter, JHEP 11 (2015) 046 [arXiv:1407.4772] [INSPIRE].

[39] A. Ochirov, Scattering amplitudes in gauge theories with and without supersymmetry, arXiv: 1409.8087 [INSPIRE].

[40] J.J.M. Carrasco, M. Chiodaroli, M. Günaydin and R. Roiban, One-loop four-point amplitudes in pure and matter-coupled $N \leq 4$ supergravity, JHEP 03 (2013) 056 [arXiv:1212.1146] [INSPIRE].

[41] M. Chiodaroli, Q. Jin and R. Roiban, Color/kinematics duality for general abelian orbifolds of $N=4$ super Yang-Mills theory, JHEP 01 (2014) 152 [arXiv:1311.3600] [INSPIRE].

[42] M. Chiodaroli, M. Günaydin, H. Johansson and R. Roiban, Scattering amplitudes in $\mathcal{N}=2$ Maxwell-Einstein and Yang-Mills/Einstein supergravity, JHEP 01 (2015) 081 [arXiv: 1408.0764] [INSPIRE].

[43] M. Chiodaroli, M. Günaydin, H. Johansson and R. Roiban, Spontaneously Broken Yang-Mills-Einstein Supergravities as Double Copies, JHEP 06 (2017) 064 [arXiv: 1511.01740] [INSPIRE].

[44] M. Chiodaroli, M. Günaydin, H. Johansson and R. Roiban, Complete construction of magical, symmetric and homogeneous $N=2$ supergravities as double copies of gauge theories, Phys. Rev. Lett. 117 (2016) 011603 [arXiv: 1512.09130] [INSPIRE].

[45] A. Anastasiou et al., Twin supergravities from Yang-Mills theory squared, Phys. Rev. D 96 (2017) 026013 [arXiv:1610.07192] [INSPIRE].

[46] M. Chiodaroli, M. Günaydin, H. Johansson and R. Roiban, Explicit Formulae for Yang-Mills-Einstein Amplitudes from the Double Copy, JHEP 07 (2017) 002 [arXiv:1703.00421] [INSPIRE].

[47] A. Anastasiou, L. Borsten, M.J. Duff, A. Marrani, S. Nagy and M. Zoccali, Are all supergravity theories Yang-Mills squared?, arXiv:1707.03234 [INSPIRE]. 
[48] M. Chiodaroli, M. Günaydin, H. Johansson and R. Roiban, Gauged supergravities and spontaneous SUSY breaking from the double copy, arXiv:1710.08796 [INSPIRE].

[49] Z. Bern and T. Dennen, A Color Dual Form for Gauge-Theory Amplitudes, Phys. Rev. Lett. 107 (2011) 081601 [arXiv:1103.0312] [InSPIRE].

[50] Z. Bern, C. Boucher-Veronneau and H. Johansson, $N \geq 4$ Supergravity Amplitudes from Gauge Theory at One Loop, Phys. Rev. D 84 (2011) 105035 [arXiv:1107.1935] [InSPIRE].

[51] Z. Bern, J.J.M. Carrasco, L.J. Dixon, H. Johansson and R. Roiban, Simplifying Multiloop Integrands and Ultraviolet Divergences of Gauge Theory and Gravity Amplitudes, Phys. Rev. D 85 (2012) 105014 [arXiv:1201.5366] [INSPIRE].

[52] Z. Bern, S. Davies, T. Dennen and Y.-t. Huang, Absence of Three-Loop Four-Point Divergences in $N=4$ Supergravity, Phys. Rev. Lett. 108 (2012) 201301 [arXiv:1202.3423] [INSPIRE].

[53] Z. Bern, S. Davies, T. Dennen and Y.-t. Huang, Ultraviolet Cancellations in Half-Maximal Supergravity as a Consequence of the Double-Copy Structure, Phys. Rev. D 86 (2012) 105014 [arXiv: 1209.2472] [INSPIRE].

[54] Z. Bern, S. Davies, T. Dennen, Y.-t. Huang and J. Nohle, Color-Kinematics Duality for Pure Yang-Mills and Gravity at One and Two Loops, Phys. Rev. D 92 (2015) 045041 [arXiv: 1303.6605] [INSPIRE].

[55] Z. Bern, S. Davies and T. Dennen, The Ultraviolet Structure of Half-Maximal Supergravity with Matter Multiplets at Two and Three Loops, Phys. Rev. D 88 (2013) 065007 [arXiv: 1305.4876] [INSPIRE].

[56] Z. Bern, S. Davies, T. Dennen, A.V. Smirnov and V.A. Smirnov, Ultraviolet Properties of $N=4$ Supergravity at Four Loops, Phys. Rev. Lett. 111 (2013) 231302 [arXiv:1309.2498] [INSPIRE].

[57] Z. Bern, S. Davies and T. Dennen, Enhanced ultraviolet cancellations in $\mathcal{N}=5$ supergravity at four loops, Phys. Rev. D 90 (2014) 105011 [arXiv:1409.3089] [InSPIRE].

[58] Z. Bern, S. Davies and T. Dennen, The Ultraviolet Critical Dimension of Half-Maximal Supergravity at Three Loops, arXiv:1412.2441 [INSPIRE].

[59] G. Yang, Color-kinematics duality and Sudakov form factor at five loops for $N=4$ supersymmetric Yang-Mills theory, Phys. Rev. Lett. 117 (2016) 271602 [arXiv:1610.02394] [INSPIRE].

[60] Z. Bern, H.-H. Chi, L. Dixon and A. Edison, Two-Loop Renormalization of Quantum Gravity Simplified, Phys. Rev. D 95 (2017) 046013 [arXiv:1701.02422] [INSPIRE].

[61] Z. Bern, A. Edison, D. Kosower and J. Parra-Martinez, Curvature-squared multiplets, evanescent effects and the $\mathrm{U}(1)$ anomaly in $N=4$ supergravity, Phys. Rev. D 96 (2017) 066004 [arXiv: 1706.01486] [INSPIRE].

[62] H. Johansson, G. Kälin and G. Mogull, Two-loop supersymmetric QCD and half-maximal supergravity amplitudes, JHEP 09 (2017) 019 [arXiv:1706.09381] [INSPIRE].

[63] Z. Bern, J.J.M. Carrasco, W.-M. Chen, H. Johansson, R. Roiban and M. Zeng, Five-loop four-point integrand of $N=8$ supergravity as a generalized double copy, Phys. Rev. D 96 (2017) 126012 [arXiv:1708.06807] [INSPIRE]. 
[64] Z. Bern, J.J. Carrasco, W.-M. Chen, H. Johansson and R. Roiban, Gravity Amplitudes as Generalized Double Copies of Gauge-Theory Amplitudes, Phys. Rev. Lett. 118 (2017) 181602 [arXiv: 1701.02519] [INSPIRE].

[65] S. Oxburgh and C.D. White, BCJ duality and the double copy in the soft limit, JHEP 02 (2013) 127 [arXiv:1210.1110] [INSPIRE].

[66] R. Saotome and R. Akhoury, Relationship Between Gravity and Gauge Scattering in the High Energy Limit, JHEP 01 (2013) 123 [arXiv:1210.8111] [INSPIRE].

[67] A. Sabio Vera, E. Serna Campillo and M.A. Vazquez-Mozo, Color-Kinematics Duality and the Regge Limit of Inelastic Amplitudes, JHEP 04 (2013) 086 [arXiv:1212.5103] [INSPIRE].

[68] S. Melville, S.G. Naculich, H.J. Schnitzer and C.D. White, Wilson line approach to gravity in the high energy limit, Phys. Rev. D 89 (2014) 025009 [arXiv: 1306.6019] [INSPIRE].

[69] H. Johansson, A. Sabio Vera, E. Serna Campillo and M.Á. Vázquez-Mozo, Color-Kinematics Duality in Multi-Regge Kinematics and Dimensional Reduction, JHEP 10 (2013) 215 [arXiv: 1307.3106] [INSPIRE].

[70] A. Luna, S. Melville, S.G. Naculich and C.D. White, Next-to-soft corrections to high energy scattering in QCD and gravity, JHEP 01 (2017) 052 [arXiv: 1611.02172] [INSPIRE].

[71] R. Monteiro and D. O'Connell, The Kinematic Algebra From the Self-Dual Sector, JHEP 07 (2011) 007 [arXiv: 1105.2565] [inSPIRE].

[72] C. Cheung and C.-H. Shen, Symmetry for Flavor-Kinematics Duality from an Action, Phys. Rev. Lett. 118 (2017) 121601 [arXiv: 1612.00868] [INSPIRE].

[73] L. Borsten, M.J. Duff, L.J. Hughes and S. Nagy, Magic Square from Yang-Mills Squared, Phys. Rev. Lett. 112 (2014) 131601 [arXiv:1301.4176] [INSPIRE].

[74] A. Anastasiou, L. Borsten, M.J. Duff, L.J. Hughes and S. Nagy, A magic pyramid of supergravities, JHEP 04 (2014) 178 [arXiv:1312.6523] [INSPIRE].

[75] A. Anastasiou, L. Borsten, M.J. Duff, L.J. Hughes and S. Nagy, Yang-Mills origin of gravitational symmetries, Phys. Rev. Lett. 113 (2014) 231606 [arXiv:1408.4434] [INSPIRE].

[76] S. Nagy, Chiral Squaring, JHEP 07 (2016) 142 [arXiv:1412.4750] [INSPIRE].

[77] A. Anastasiou, L. Borsten, M.J. Hughes and S. Nagy, Global symmetries of Yang-Mills squared in various dimensions, JHEP 01 (2016) 148 [arXiv: 1502.05359] [INSPIRE].

[78] G.L. Cardoso, S. Nagy and S. Nampuri, A double copy for $\mathcal{N}=2$ supergravity: a linearised tale told on-shell, JHEP 10 (2016) 127 [arXiv:1609.05022] [INSPIRE].

[79] G. Cardoso, S. Nagy and S. Nampuri, Multi-centered $\mathcal{N}=2$ BPS black holes: a double copy description, JHEP 04 (2017) 037 [arXiv:1611.04409] [INSPIRE].

[80] B.R. Holstein and J.F. Donoghue, Classical physics and quantum loops, Phys. Rev. Lett. 93 (2004) 201602 [hep-th/0405239] [INSPIRE].

[81] F. Bloch and A. Nordsieck, Note on the Radiation Field of the electron, Phys. Rev. 52 (1937) 54 [INSPIRE].

[82] T. Kinoshita, Mass singularities of Feynman amplitudes, J. Math. Phys. 3 (1962) 650 [INSPIRE].

[83] T.D. Lee and M. Nauenberg, Degenerate Systems and Mass Singularities, Phys. Rev. 133 (1964) B1549 [INSPIRE]. 
[84] E. Laenen, G. Stavenga and C.D. White, Path integral approach to eikonal and next-to-eikonal exponentiation, JHEP 03 (2009) 054 [arXiv: 0811.2067] [INSPIRE].

[85] C.D. White, Factorization Properties of Soft Graviton Amplitudes, JHEP 05 (2011) 060 [arXiv: 1103.2981] [INSPIRE].

[86] Z. Bern and D.A. Kosower, Efficient calculation of one loop QCD amplitudes, Phys. Rev. Lett. 66 (1991) 1669 [INSPIRE].

[87] Z. Bern and D.A. Kosower, The Computation of loop amplitudes in gauge theories, Nucl. Phys. B 379 (1992) 451 [INSPIRE].

[88] M.J. Strassler, Field theory without Feynman diagrams: One loop effective actions, Nucl. Phys. B 385 (1992) 145 [hep-ph/9205205] [INSPIRE].

[89] J. Vines, Scattering of two spinning black holes in post-Minkowskian gravity, to all orders in spin and effective-one-body mappings, arXiv:1709.06016 [INSPIRE].

[90] Z. Bern and A.K. Grant, Perturbative gravity from QCD amplitudes, Phys. Lett. B 457 (1999) 23 [hep-th/9904026] [INSPIRE].

[91] C. Cheung and G.N. Remmen, Twofold Symmetries of the Pure Gravity Action, JHEP 01 (2017) 104 [arXiv: 1612.03927] [INSPIRE].

[92] C. Cheung and G.N. Remmen, Hidden Simplicity of the Gravity Action, JHEP 09 (2017) 002 [arXiv: 1705. 00626] [INSPIRE]. 